$$
\text { "konya" — 2015/5/22 — 10:17 — page } 121 \text { — \#1 }
$$

\title{
The formation of area concept with the help of manipulative activities
}

\author{
ESZTER HERENDINÉ-KÓNYA
}

\begin{abstract}
Examining the performance of Hungarian students of Grades 4-12 in connection with area measurement, we found many deficiencies and thinking failures. In the light of this background, it seems reasonable to review the educational practice and to identify those teaching movements that trigger the explored problems and to design a teaching experiment that tries to avoid and exclude them.Based on result we make recommendations for the broad teaching practice. In our study we report on one part of a multi-stage teaching experiment in which we dealt with the comparison of the areas of figures, the decomposition of figures and the special role of the rectangle in the process of area concept formation. The conclusion of the post-test is that manipulative activities are important and necessary in Grades 5 and 6, more types of equidecomposition activities are needed and the number of measuring tasks with grid as a tool should also be increased.
\end{abstract}

Key words and phrases: area measurement, manipulative activities, concept formation.

ZDM Subject Classification: D73, G33, U63.

\section{Introduction}

Examining the performance of Hungarian students of Grades 4-12 in connection with area measurement, we found many deficiencies and thinking failures.

Students are often capable to solve only tasks which require the use of simple formulas, and very often we experience the nonsense use of the learnt formulas. When defining the area of irregular 2-dimensional figures they are not able to use the additive property of the area, they do not see the parts in a compound figure, 


$$
\text { "konya" — 2015/5/22 — 10:17 — page } 122 \text { — \#2 }
$$

which area could be easily calculated using one of the learnt formulas. They have little knowledge and experience about the conservation of the area, that is, not only congruent shapes have the same area. The well-known area formula of the rectangle makes them generalise wrongly that the area of any kind of polygon can be calculated from the length of the sides [9]. Besides mixing up the concepts of area and perimeter, the confusion of length- and area measures also refers to the fact that students from different grades do not have a stable conceptual background which would help them to concentrate on more complex tasks in this topic besides the simple routine exercises, and to solve them successfully.

In the Hungarian teaching practice the following phenomena can be observed:

- The concept of area is too early narrowed down to the area of rectangle and to its calculation formulas.

- In most of the area-determination tasks we ask to measure the length of the sides (rectangle, right-angled triangle, right-angled trapezoid).

- Perimeter and area are taught simultaneously. In addition to that, the sounding of the two words are similar in Hungarian.

- Instead of manual activities, drawing exercises are dominant in the primary classes. These tasks include oversimplified tessellation and equidecomposition situations.

- We do not seek explanation for the question that from all suitable tiles why the square became the standard unit of the area.

- The experience about the area conservation is very little, so it doesn't countervail the wrong conclusions from stories that the bigger the perimeter of a figure, the bigger its area.

In Hungary, teaching measurement in primary school has a well-developed criteria system, the measuring of length, weight and capacity is taught according to it. First, quantities are compared directly without any measuring, and then follow the indirect comparison, the introduction of the concept of measure and the understanding the significance of measuring. At the beginning we choose arbitrary units. During the measuring practices we gain experiences about the necessary inaccuracies of the measurement and about the relationship between the size of the unit and number required to measure. The smaller unit we use, the more accurate the measure will be, however, the appropriate unit can be found for each quantity. Measuring the same quantity with a bigger unit we get a smaller number (inverse proportionality), while measuring a bigger quantity with the same unit will result in bigger number (direct proportionality). The studying 


$$
\text { "konya" — 2015/5/22 — 10:17 — page } 123 \text { — \#3 }
$$

of standard units and the conversions between them happens only after getting the above mentioned measuring experiences.

Estimation also has a very important role. The estimation competence is crucial in our everyday life, but it also helps in solving mathematical problems if we can decide on the value of a result: real or not. The estimation competence can be developed by the frequent repetition of the following three activities: estimation, measuring, comparison of the estimated and measured values [8]. While the Hungarian curriculum and the teaching practice tries to follow the above mentioned general principles when teaching the measuring of length, weight and capacity in the primary classes, the measurement of area and volume is taught in a different way: derived from the length.

In the light of this background, it seems reasonable to review the educational practice and to identify those teaching movements that trigger the explored problems and to design a teaching experiment that tries to avoid and exclude them.

In our study we report on one part of a multi-stage teaching experiment in which we dealt with the comparison of the areas of figures, the decomposition of figures and the special role of the rectangle. Finally, based on results, we make recommendations for the broad teaching practice.

\section{Theoretical background}

The possible causes of the conceptual confusion connected to the concept of area have been dealt with by a number of studies.

Some studies point out that in order to define the area, the measurement should be done directly and not indirectly, measuring the lengths and calculating the area $([14],[15],[19])$. In the initial phases of the concept formation it can be useful to consider area as a quantity independent from length.

Curry, Mitchelmore and Outhred lists five principles, which are the following: "1. the need for repeated units that do not change; 2. the appropriateness of a selected unit; 3. the need for the same unit to be used to compare two or more objects; 4. the relationship between the size of the unit and the number required to measure; 5. the structure of the repeated units." [6]. The study analyses the measuring of length, area and volume independent from each other and according to the listed principles.

Baturo and Nason in [5] describes the gist of measurement as continuous quantities being divided into equal discrete units and then counted. The measuring can be done in two ways: we take a unit and cover the whole quantity 


$$
\text { "konya" — 2015/5/22 — 10:17 — page } 124 \text { — \#4 }
$$

successively, or we take the required size unit and cover the whole quantity at once. Taking into consideration the nature of area measurement, only the latter approach can be used.

The formulas of area calculation are introduced too early, long before a stable concept image [18] would be formed in the students' minds. If the calculation rules are not linked to actual experiences, the knowledge of area is not effective [5]. The early use of formula focuses on the static features of the area, so there is no chance to experience the dynamic features that would make the matching of different figures' area possible.

In understanding area measurement, area conservation has a crucial role, that is, the fact that the area of a figure doesn't change if the figure is cut up and a new figure is composed from the parts [17]. According to Kordaki [12], area conservation, area measurement and area formulas should be taught in an integrated way, in order to develop all three aspects. The experiment of Kamii and Kysh shows that students claim that the area of the new figure created by the reorganisation of the parts is not the same as the area of the original figure [11]. The study also shows that the type of the figures may have a role in recognising the area conservation: in the case of parallelogram students accept it more easily than in the case of cutting up triangles or irregular figures.

Kospentaris et al. in [13] claims that recognising area conservation could cause problems for secondary school students and even for first year university students. In the lack of mathematical basics which is necessary for geometric reasoning, students rely on intuitive experiences and/or visual recognition and give wrong answers for questions regarding area conservation. The idea that only the areas of congruent figures are equal is very strongly rooted.

In the study of mathematical thinking there is a well-known principle: "the bigger A, the bigger B" [19]. In our study this principle appears in the exclusive use of the following rule: "the bigger the perimeter of a figure, the bigger its area". By studying the relationship between the perimeter and the area, experiences can be gained about the conservation of the area, and about the dynamic nature of area concept ([3], [4], [5]).

In Zacharos' paper [20] we can read about the teaching practice of area measurement and the mistakes of concept formation. He sees the problem in the too early introduction of formulas, but also refers to the misunderstandings which roots from the wording. Unlike the word 'length', the 'area' is not used in the same way in our everyday life as in mathematics. Area is not only used to denote a measure, the quantity describing a plane figure, but very often by the word 


$$
\text { "konya" — 2015/5/22 - 10:17 — page } 125-\# 5
$$

'area' we mean the domain itself, and it can also occur that it means the multiplication of the width and length as it could be linked to the rectangular figure. In literature or everyday texts it often refers to the rectangle, but it is not made clear, so it strengthens the wrong idea that the quantity of area only could mean the area of rectangle. "... measure out this area by steps, which is surrounded by the blood of our judge,..." (Mikszáth: A fekete város) The previously mentioned "bigger perimeter, bigger area" principle is referred to in the following example: "As much as you can go round on your feet in a day is yours" (Tolsztoi: How much land does a man need?)

The cited studies confirm that for the introduction of area measuring practices is needed which are independent from length. For development of the teaching plan of area measurement we applied the general principles of measurement teaching $[8]$ in a way that in the beginning of the development we considered area as an independent quantity from length.

When posing problems for the area measurement we can ignore the representation theory of Brunner. Brunner suggested three ways of transforming experiences into a model of the world: the enactive, the iconic and the symbolic representation (as cited in [1], p. 37). In our teaching experiment we concentrate first on the enactive (concrete actions with concrete objects) then the iconic (pictures and imagined situations) representation.

Tessellation with congruent two-dimensional figures is the activity with which an area of a figure can be measured. Tessellation means to cover the plane without gaps and overlaps. The choice of suitable unit of area measurement, that is tile, is not an easy task for students and it is hard to understand why even the square became the standard unit of area [20]. Experience shows that the shape of the figure has an influence on the choice of the unit [7]. The wrong intuition can be observed, that a figure can be covered with similar congruent tiles. If the choice of the suitable tile is successful, the next task is to organise them in the right way in order to cover the figure. It can also occur that students fill in the remaining gaps with other tiles [15]. It also means a difficulty to find the fraction of the chosen tile, unlike e.g. in the case of length measurement.

The mathematical concept of the area gives opportunity for two kinds of manual activities, namely the tiling (tessellation) and equidecomposition.

By the area of a two-dimensional figure we mean a positive real number for which the followings are true: a) the areas of congruent figures are equal; b) the sum of the area of subfigures is equal to the area of the whole figure; c) the unit side square's area is $1[16]$. 


$$
\text { "konya" — 2015/5/22 — 10:17 — page } 126 \text { — \#6 }
$$

The Wallace-Bolyai-Gerwien theorem about the equidecomposition of polygons describes the significant role of the decomposition into a rectangle: Any two simple polygons of equal area are equidecomposable.

The theorem can be proven by the following steps: a) Any triangle can be converted to a rectangle; b) two parallelograms with same width and height can be converted to each other; c) any rectangle can be decomposed into a rectangle with a side of a given length. Consequently, it follows that every polygon can be decomposed into a rectangle. The rectangles can be covered with suitably chosen squares, so we get an explanation why it is reasonable to choose the square as the measure of the area. The decomposition into rectangle shows that in the definition of the area of the polygon, rectangle gains a significant role. Indeed, each area formula can be backed to the area formula of the rectangle.

\section{Research question}

From the above described two types of manipulative activities this study deals with the equidecomposition.

Questions:

(1) What kind of equidecomposition, area measurement activities are worth to use during the formation and consolidation of the area concept?

(2) What are the problems and challenges of these activities?

\section{Methodology}

In the Grades 4, 5 and 6 we made preliminary observations and planned a teaching experiment including 5 lessons (45 minutes normal classes) focusing on equidecomposition and area measurement activities.

We ran the teaching experiment in one of the classes of the practicing school of the teacher training college. The school is a highly selective lower secondary school taking the best achieving students from the city with good social background.

The first 3 lessons (1-3) were held in the $5^{\text {th }}$ Grade (Class 5c.) in spring, 2012, in 3 consecutive weeks. Students already had some information about tessellation and about covering with unit squares. They have already learnt the calculation methods of the area of the rectangle and the square. 


$$
\text { "konya" — 2015/5/22 — 10:17 — page } 127 \text { — \#7 }
$$

During the lessons, students worked in 9 groups, including 3-4 members. Problems are presented to the students then they are given time to think on their own about possible answers for a specific amount of time. Finally, groups discuss their answers in the classroom. We apply cooperative teaching technique [10], which are rarely used in Hungarian school mathematics teaching [2]. In contrast our students were familiar with this method; their teacher was expert in organization of students into groups and in supervision of the classroom work.

The $4^{\text {th }}$ and $5^{\text {th }}$ lessons were held in the same class (Class 6c.), a year later, in spring, 2013, once in a week for 2 weeks. Before this lessons the students haven't dealt with the area measurement, this topic came up again at the end of the year, only after our teaching experiment. The area formulas of special figures were introduced only then: parallelogram, triangle, trapezoid and deltoid.

During the experiment 29 students worked independently, they recorded their answers on task sheets.

All the 5 lessons were voice-recorded, notes and photos were taken.

In the week following the lessons $6^{\text {th }}$ Grade students had to write a post-test, as well as a control group of $7^{\text {th }}$ Grade students who haven't dealt with the area measurement tasks in that year.

The material of the lessons:

Lessons 1-2: Manual activities for comparing the area of figures without actual measuring, using equidecomposition if necessary.

Lesson 3: Manual activities for converting figures into rectangles, and the tessellation of these rectangles using squares

Lesson 4: Refreshing the activities from the previous year; comparing the area of figures

Lesson 5: Using square grid as a measurement tool, determining the area of figures after converting them to rectangle with the use of square grid.

Post-test: determining the area of rectangles and parallelograms of different shape and position with square grid and/or calculation with length measurement.

\section{Description of the activities and results}

Lessons 1-2: Comparing the area of figures

Aim: to deepen the area concept with direct quantity comparisons; to gain experience about the existence of non-congruent figures that have the same area; to get knowledge about the equidecomposition activity and to learn how to use 


$$
\text { "konya" — 2015/5/22 — 10:17 — page } 128 \text { — \#8 }
$$

it for the comparison of the area of figures; to learn about the simple process of converting figures into rectangle.

\section{Experiences}

Task 1: Comparing the area of two figures cut from a newspaper, an L-shape and a cross-shape

Previously we asked students to give ideas for the comparison of the areas of the figures:

S1: "We put the L-shape to the cross and cut the floppy ends."

S2: "Try to cover the cross with the same shape that L could be covered with."

It can be seen that students know two ways for comparing the areas: direct comparison by placing the figures onto each other; indirect comparison by choosing a suitable unit of the measurement.

While using the direct comparison in the group work, there were groups which cut the L-shape and other groups that cut the cross-shape. In both cases they defined the relationship of the areas well.

Task 2: Comparing the areas of three rectangles

The sides of the given rectangles were different, but using one cut on each rectangles, they could be converted into congruent ones. Each group could identify the congruency of the figures (Figure 1).

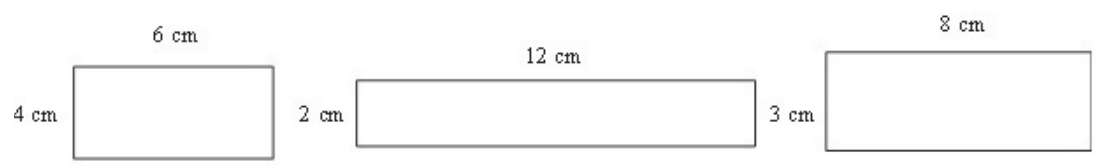

Figure 1

Analysing the ways of the comparisons it can be observed to what extent students relied on the transitive properties of the relation:

- 6 groups out of 9 converted two rectangles into a third $(A \rightarrow C, B \rightarrow C)$, that is, they recognised that things which equal to the same thing also equal to each other (the symmetrical and transitive properties of equality).

- Another group used the transitivity but not the symmetry, because they converted two rectangles into each other, and a third one into one of them.

- 2 groups converted each rectangle into another $(A \rightarrow B, B \rightarrow C, C \rightarrow A)$, so they didn't use the transitivity of ordering. 


$$
\text { "konya" — 2015/5/22 — 10:17 — page } 129 \text { — \#9 }
$$

Task 3: Comparing the areas of a rectangle, a right-angled trapezoid and a symmetrical trapezoid.

We chose two trapezoids with a size that can easily be converted into rectangles with sides of $3 \mathrm{~cm}$ or $6 \mathrm{~cm}$ (Figure 2), so the rectangles after the equidecomposition can be compared directly.
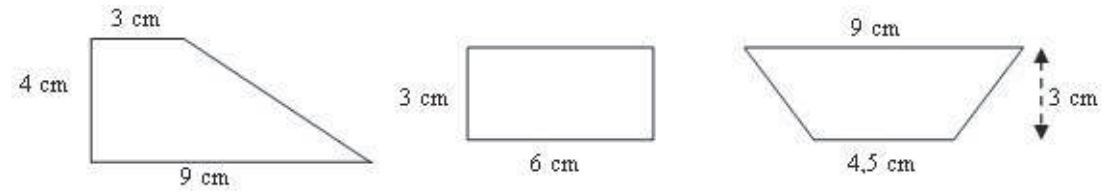

Figure 2

- Each group identified the relationship between the areas well $(B<C<A)$.

- 7 groups converted both trapezoids into rectangles, but only 1 group got rectangles which have one congruent side $(3 \mathrm{~cm})$. The others converted the right-angled trapezoid to a rectangle of sides $4 \mathrm{~cm}$ and $6 \mathrm{~cm}$, and the symmetrical trapezoid to a rectangle of sides $3 \mathrm{~cm}$ and $6.75 \mathrm{~cm}$, so they could only conclude that the area of the rectangle is the least (Figure 3). They determined the relationship between the areas of trapezoids by visual estimation after converting them into rectangles and placing them onto each other.

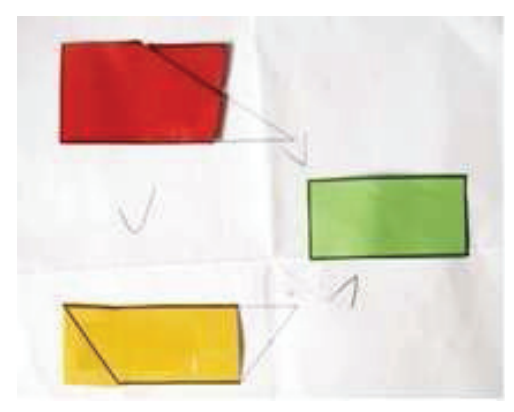

Figure 3 
- 1 group wanted to convert the two trapezoids into the given rectangle by cutting down the unnecessary parts. Obviously, from the right-angled trapezoid more parts ("larger area") had to be cut, they considered it as the figure which area is the larger.

- 1 group couldn't convert the symmetrical trapezoid into a rectangle, they used only visual estimation.

Task 4: Justification of the given relation between the areas of a cross-shape and a right-angled triangle using equidecomposition (Figure 4)
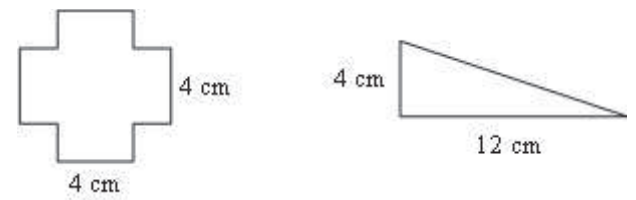

Figure 4

- 6 groups cut a rectangle from the cross-shape then placed the triangle onto it and claimed that the area of the cross-shape is twice as big as the area of the triangle.

S3: "The statement is true, because if we cut the two sides of the cross and place it under the standing rectangle, then the triangle fits into it twice."

- 2 groups cut the cross-shape in the same way, but they converted the triangle into a rectangle cutting it by its midline.

- The work of one group cannot be evaluated, because they tried to cut the rectangle and place it somehow on the cross-shape.

The solutions of the tasks show that:

- The comparison in some cases can be done by visualisation, equidecomposition is not necessary.

- The cutting up of the figures was unusual at the beginning; before measuring of other quantities the quantities are not converted.

- The cuts used in the equidecomposition in the given cases are natural, but they do not necessarily prepare the formulas.

- Students use the additive properties of the area in the right way, but they do not really feel the significance of converting into rectangle. 
- Not everyone uses the symmetrical and transitive properties of the equality and order relations during the comparison.

Lesson 3: Tessellation with squares and equidecomposition to rectangle

Aim: to introduce square as the unit of the area; to learn the area formula of rectangle by experience.

Based on the experience from the previous tasks, the goal now was to determine the area of the right-angled triangle and the parallelogram by converting them into a rectangle (Figure 5).
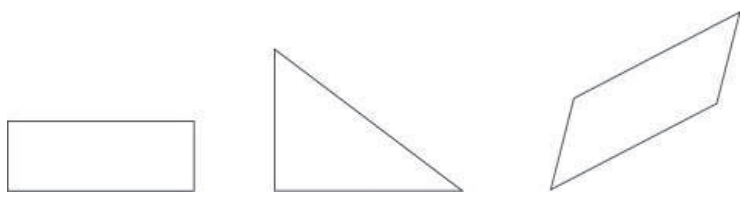

Figure 5

\section{Experiences}

- All 9 groups could successfully convert the parallelogram into a rectangle.

- 8 groups could solve the conversion of the triangle; 7 groups cut the triangle by the midline parallel with the leg, and 1 group cut it by the midline and by the height belonging to the hypotenuse.

- 7 groups created two rectangles congruent with the original one. 5 out of all groups covered only the original one with squares (Figure 6a), and two made a grid, so they created a tool applicable for area measurement (Figure 6b).

- 2 groups converted the shapes into non-congruent rectangles. They put the squares along the sides only and used the multiplication rule (Figure 6c).

The solutions of the tasks show that:

- Some students discovered the grid, which is a suitable measuring tool of area measurement.

- Some students put the squares along the sides, which means that they simplified the enumeration of squares and so discovered the rectangle's area formula. 


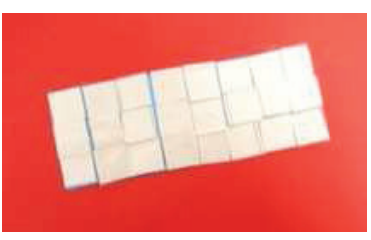

(a)

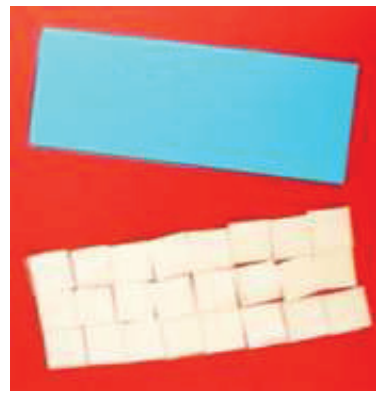

(b)

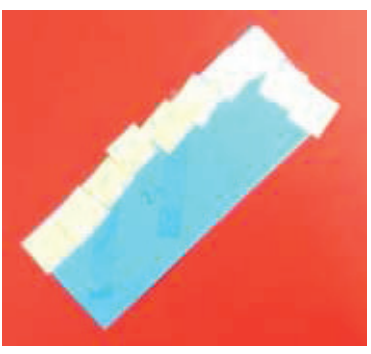

(c)

Figure 6

Lesson 4: Comparing the area of figures - 1 year later

Aim: to refresh what was learnt in the previous year; to practice the conversion into rectangle.

\section{Experiences}

Task 1: to compare the area of figures $A$ and $B$, those were cut out from paper (Figure 7).

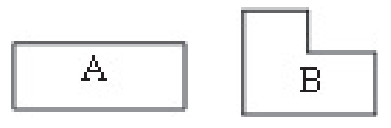

Figure 7

The L-shape was converted to a rectangle by 14 students, the rectangle to an L-shape by 13 students, and both figures were cut into congruent rectangles by 2 students. Examples for the justification of the equality of the areas:

S4: "The difference is only between the shapes of the plane figures. If we cut down a little piece from $B$ and paste together with the other piece, it will be identical with A." - That is, if one figure is equidecomposable to the other, their areas will be equal.

S5: "The floppy part fits to the floppy part of B."

Task 2: to compare the area of figures $C$ and $D$, those were cut out from paper (Figure 8). 


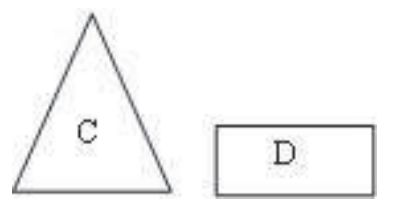

Figure 8

- 28 students determined well, that $C>D$, and according to 1 student $C=D$.

- 19 students cut the triangle:

- Rectangle was created by 5 students.

S6: "If I cut $C$ into two halves and put the pieces together in a rectangle shape, than $C$ rectangle will be bigger than $D$ rectangle."

- 1 student put the halves of the triangle wrongly (Figure 9a).

- 13 students cut the triangle into 3-4 pieces and claimed that not all pieces fit to the rectangle.

S\%: "If we cut $C$ into pieces and try to assemble $D$ from the pieces, then some pieces will still be left from."

- Out of the 13 students, 2 cut the triangle in a way that could make it suitable to create the general area formula of the triangle (Figure $9 \mathrm{~b}$ ) S8: "I measured $C$ onto $D$ and I cut the pieces which was too much. I halved what was left and put it on D."

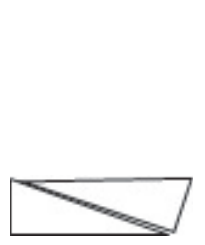

(a)

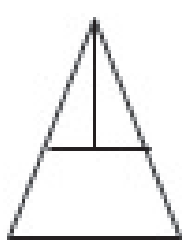

(b)

Figure 9

- The rectangle was converted into triangle by 10 students.

S9: "If we put $D$ onto $C$, it can be seen that $D$ cannot cover it, howsoever we would try to cut it."

Task 3: to compare of the area of two rectilinearpolygons with grid (Figure $10)$. 


$$
\text { "konya" — 2015/5/22 — 10:17 — page } 134 \text { — \#14 }
$$

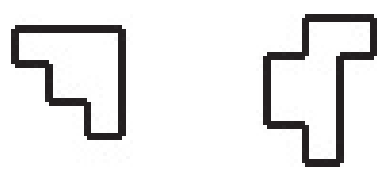

Figure 10

Everyone considered the area of the right figure larger, they compared the numbers of the small squares.

S10: "The one had the bigger area in which more squares were."

The solutions show that:

- Activities that were practiced formerly will remain in students' memory. Comparing areas with equidecomposition doesn't cause problems.

- Conversion into rectangle wasn't preferred by the students, though comparison tasks didn't motivate them to do so.

- The use of grid as a measurement tool is known for the students.

Lesson 5: Determining the area of figures after converting them to rectangles using grid

Aim: to measure area using grid; to determine the area of right-angled triangle, parallelogram, and rectilinear figures. (The figures can be touched and equidecomposed.)

\section{Experiences}

Students were successful in the conversion of well-known figures into rectangles and used the grid in the right way when determining their area.

\section{Post-test}

The test was given to the students on papers; this time they had no opportunity to do tessellation or equidecomposition.

The tasks were solved by the experimental class ( $6 c$; 32 students) and by a control class ( $\%$ c; 20 students).

In the $1^{\text {st }}$ task they were asked to determine the area of 4 rectangles with different shape and position (Figure 11)

The method was not predetermined and the students got tools: $\mathrm{cm}^{2}$-grid and ruler. The position of $A, B$ and $C$ rectangles are usual, that is the sides are 


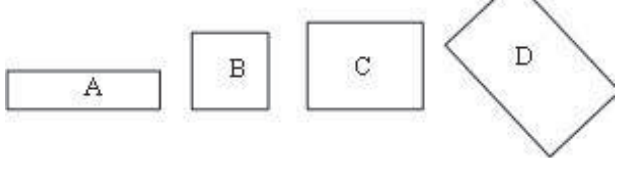

Figure 11

parallel with the edges of the paper. Putting $\mathrm{cm}^{2}$-grid properly to the $A, B$ and $D$ rectangles, whole squares should be counted, while in the case of $C$ rectangle there will be 6 "half squares" too.

In the $2^{\text {nd }}$ task we asked to determine the area of 5 parallelograms with different shape and position (Figure 12) by converting them to rectangles and then using grid and/or calculation.

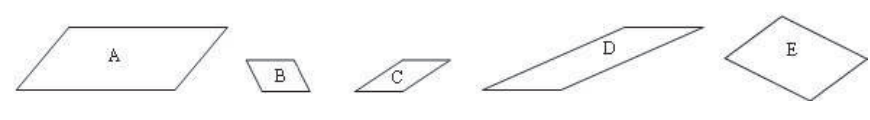

Figure 12

The conversion into rectangle is harder in the case of $C, D$ and $E$ parallelograms because their shape and position makes it difficult to find the right cut-line (height).

\section{Results and discussion}

\section{Task 1}

26 students gave the right answer from the experimental class $(6 c$.) for the area of the four rectangles, while from the control class $(7 z)$.13 right answers were given.

The other 6 students from the experimental class have the right conceptual knowledge too, but either because the position of the $D$ rectangle or calculation error they didn't get the right results.

In class $7 z, 4$ students calculated with the perimeter formula and 3 with the formula " $a \cdot b \cdot 2$ ". It also happened, that with the grid they got the right solutions, but not with the calculation. The area formula of the rectangle was already known by the students in both classes in the examined period, but students in the $6^{\text {th }}$ 
class practiced it less and used it a longer time ago which probably was the reason that the two methods did not contradict.

It can be concluded that all students in the class $6 c$. have the right area concept, at least in the case of rectangles.

The visual representation of the area concept was missing in many students in the $7^{\text {th }}$ class, there was no content behind the formula.

\section{Task 2}

In order to determine the area of the parallelograms in the $2^{\text {nd }}$ task we recommended two kinds of methods using the tools. We asked students to start the solution with conversion to rectangle then determine the area of the rectangles using grid or measuring lengths.

In both classes half of the students (16 and 10, respectively) could convert all of the parallelograms to rectangle and by using the grid or measuring the sides could determine the areas.

9 students from $6 c$. and 4 students from $7 z$. couldn't convert all parallelograms into rectangles, so they didn't get results in all cases.

In class $6 c .4$ students tried to use the grid without converting the figures into rectangles and 2 students used the formula " $a \cdot b$ " when the conversion was not successful; 1 student didn't produce results that could be evaluated.

In class $7 z .2$ students calculated by using the perimeter formula after converting the parallelogram into rectangle, and 3 students each at least in one case used the formula " $a \cdot b$ " when they couldn't convert the figure. 1 student used the correct calculating method for the area of the parallelogram, what they learned in the previous year.

The results clearly show the mistakes resulting from the early formula use: in those cases where students couldn't convert to rectangle, the younger ones applied the grid and approximation, so they used the correct concept image of the area in the right way, while the older ones seceded from the conceptual picture and 5 of them calculated using the wrong formula.

Analysing the solutions of the two tasks students could be categorised according to the following:

(1) In both tasks they could determine the area of each figure. In cases when both methods were used, they got the same results.

(2) They couldn't determine the area of those figures which position was different from the usual, but didn't give wrong results.

(3) At least in one case they calculated with the perimeter formula. 
(4) The area of some parallelograms was given by the multiplication of the sides.

(5) One of the rectangles' area was calculated by the $a b 2$ formula.

(6) More mistakes were made from type 3, 4 and 5. (Figure 13)

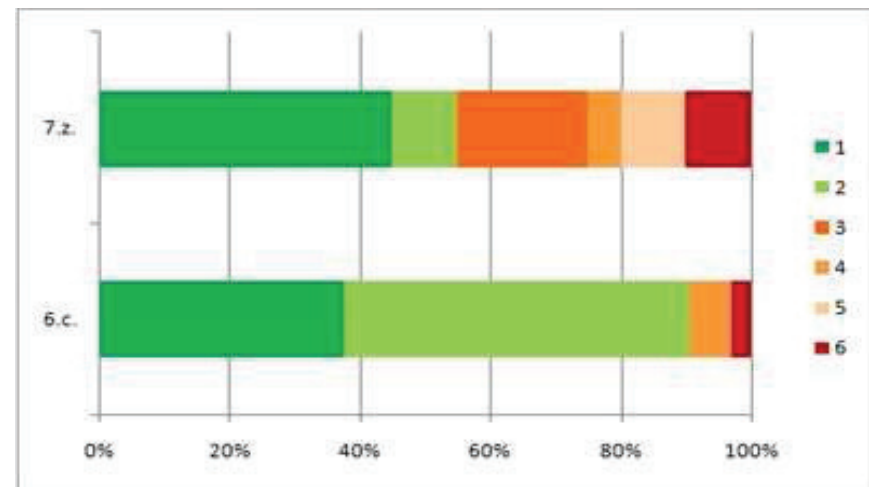

Figure 13

The diagram clearly shows that more than $90 \%$ of the experimental class students didn't make conceptual mistakes ( $1^{\text {st }}$ or $2^{\text {nd }}$ type solution), while this ratio is smaller in the control group, less than $60 \%$. The wrong use of formulas was also more typical in this class. Most of the time they tried to give the area by measuring length than calculation, instead of using the grid.

\section{Conclusion}

The recent study highlights that manipulative activities are important and necessary.

Equidecomposition activities should be divided two different groups: 1. arbitrary conversion of figures in order to compare areas; 2 . conversion of figures into rectangles.

It could be useful to do equidecomposition activities with figures that are more difficult to compare.

The afterimages of activities are better preserved by the long term memory than the content of correctly deduced formulas. 


$$
\text { "konya" — 2015/5/22 — 10:17 — page } 138 \text { — \#18 }
$$

Although the teaching experiment was organized in a special, highly selected school, the results i.e. the identified problems allow us to make some recommendations for the broad teaching practice:

The above detailed activities should be practiced already in the Grade 5 and they should precede the introduction of the area formulas of parallelogram, triangle, trapezoid and deltoid. It would be reasonable to start with comparison and equidecomposition, gradually proceed to the conversion to rectangle.

It would also be important to use several types of figures that are more difficult to convert.

In reality equidecomposition is executed through many tasks because it is difficult to abstract from the drawn position of the figure.

The introduction of the formulas cannot happen until the area measurement with the grid becomes a routine.

\section{References}

[1] A. Ambrus, Bevezetés a matematikadidaktikába, ELTE Eötvös Kiadó, Budapest, 1995 (in Hungarian).

[2] A. Ambrus and K. Barczi-Veres, Using open problems and cooperative methods in mathematics education, LUMAT 3, no. 1 (2015), 3-18.

[3] R. Babai, T. Levyadum and D. Tirosh, Intuitive rules in science and mathematics: A reaction time study, International Journal of Mathematical Education in Science and Technology 37 (2006), 913-924.

[4] R. Babai, H. Zilber, R. Stavy and D. Tirosh, The effect of intervention on accuracy of students' responses and reaction times to geometry problems, International Journal of Mathematical Education 8 (2010), 185-201.

[5] A. Baturo and R. Nason, Student teachers' subject matter knowledge within the domain of area measurement, Educational Studies in Mathematics 31, no. 3 (1996), 235-268.

[6] M. Curry, M. Mitchelmore and L. Outhred, Development of children's understanding of length, area and volume measurement principles, no. 2, Proceedings 30th Conference of the International Group for the Psychology of Mathematics Education Praha, Czech Republic, (J. Novotná, H. Moraová, M. Krátká and N. Stehlíková, eds.), 2006, 377-384.

[7] B. Heraud, Conceptions of area units by 8-9 year old children, no. 3, Proceedings 9th Conference of the International Group for the Psychology of Mathematics Education Montreal, Canada, (J. Bergeron, ed.), 1987, 299-304.

[8] E. Herendiné Kónya, A méréstanítás általános szempontjai, A matematika tanítása az alsó tagozaton, (E. Herendiné Kónya, ed.), Nemzedékek Tudása Tankönyvkiadó, Budapest, 2013, 240-248 (in Hungarian). 
[9] E. Herendiné Kónya, How can high school students solve problems based on the concept of area measurement?, Proceedings of the 15th ProMath Conference $30 \mathrm{Au}-$ gust-1 September 2013 in Eger, (A. Ambrus and É. Vásárhelyi, eds.), Mathematics Teaching and Education Center, 2014, 95-107.

[10] S. Kagan, Kooperativ tanulás, ÖKONET, Budapest, 2001 (in Hungarian).

[11] C. Kamii and J. Kysh, The difficulty of "length $x$ with": Is a square the unit of measurement?, Journal of Mathematical Behavior 25 (2006), 105-115.

[12] M. Kordaki, The effect of tools of a computer microworld on students' strategies regarding the concept of conservation of area, Educational Studies in Mathematics 52, no. 2 (2003), 177-209.

[13] G. Kospentaris, P. Spyrou and D. Lappas, Exploring students' strategies in area conservation geometrical tasks, Educational Studies in Mathematics 77, no. 1 (2011), $105-127$.

[14] C. Murphy, The role of subject knowledge in primary student teachers' approaches to teaching the topic of area, Proceedings of CERME6, January 28th-February 1st 2009 Lyon, (France), (V. Durand-Guerrier, S. Soury-Lavergne and F. Arzarello, eds.), Institut National de Recherche Pédagogique, 2010, 1821-1830.

[15] E. Nitabach and R. Lehrer, Developing spatial sense through area measurement, Teaching Children Mathematics 2, no. 8 (1996), 473-476.

[16] Matematika az általános képzéshez a tanító szak számára, (Gy. Pappné Ádám, ed.), Nemzeti Tankönyvkiadó, Budapest, 1996 (in Hungarian).

[17] J. Piaget, B. Inheldr and A. Seminska, The child's conception of geometry, Routledge and Kegan Paul, London, 1960.

[18] D. Tall and S. Vinner, Concept image and concept definition in mathematics with particular reference to limits and continuity, Educational Studies in Mathematics 12, no. 2 (1981), 151-169.

[19] P. Tsamir, Using the intuitive rule more A - more B for predicting and analyzing student's responses in geometry, International Journal of Mathematical Education in Science and Technology 34, no. 5 (2003), 639-650.

[20] K. Zacharos, Prevailing educational practices for area measurement and students' failure in measuring areas, Journal of Mathematical Behavior 25 (2006), 224-239.

ESZTER HERENDINÉ-KÓNYA

UNIVERSITY OF DEBRECEN

DEBRECEN

HUNGARY

E-mail: eszter.konya@science.unideb.hu

(Received March, 2015) 